\title{
Effects of exercise on lymphocytes and cytokines
}

\author{
Bente Klarlund Pedersen, Anders Dyhr Toft
}

\begin{abstract}
Objectives-To review results on exercise induced changes in the immune system following strenuous and moderate exercise. Methods-A literature search over the past 15 years was conducted using Medline and selected papers.

Results-After intense long term exercise, the immune system is characterised by concomitant impairment of the cellular immune system and increased inflammation. Thus low concentrations of lymphocytes, suppressed natural immunity, suppressed lymphocyte proliferation, and suppressed levels of secretory IgA in saliva are found simultaneously with high levels of circulating proinflammatory and antiinflammatory cytokines. The underlying mechanisms are multifactorial and include neuroendocrinological and metabolic factors. The clinical consequences of the exercise induced immune changes have not formally been identified, but the exercise effect on lymphocyte dynamics and immune function may be linked to the exercise effects on resistance to infections and malignancy and the cytokine response may be linked to muscle damage or muscle cell growth.
\end{abstract}

Conclusions-Moderate exercise across the life span seems to increase resistance to upper respiratory tract infections, whereas repeated strenuous exercise suppresses immune function. It is premature to offer advice on nutrition to athletes in order to alter the exercise induced immunosuppression found after exercise.

(Br F Sports Med 2000;34:246-251)

The Copenhagen Muscle Research

Centre and

Department of

Infectious Diseases

Rigshospitalet,

University of

Copenhagen, Denmark

B K Pedersen

A D Toft

Correspondence to: Dr B K Pedersen,

Department of Infectious

Diseases M7721,

Rigshospitalet, Tagensvej 20,

2200 Copenhagen $\mathrm{N}$,

Denmark

email: bkp@rh.dk

Accepted for publication 9 March 2000

Epidemiological evidence exists that supports the anecdotal impression ${ }^{1}$ that regular exercise increases resistance to infections such as the common cold, whereas hard training is associated with increased upper respiratory tract infections. ${ }^{23}$ Also, there is accumulating evidence that exercise is a lifestyle that offers some protection against malignancy. ${ }^{4}$ It has become clear that moderate exercise stimulates the immune system and may be somewhat responsible for exercise related reduction in illness. However, strenuous exercise induces immunosuppression in the recovery period and may explain the increased risk of infection in athletes. $^{56}$

Interest in the effect of physical activity on the immune system is not limited to exercise physiologists. It is a valid aim that results from studies on exercise immunology can be integrated into understanding immunological processes in clinical medicine. Furthermore, results from the field of exercise immunology may help to guide athletes and contribute to public health recommendations on exercise and infections.

This article provides a review of various aspects of exercise immunology. Effects of acute and chronic exercise on lymphocyte function and cytokine levels are described and this is followed by a discussion of the clinical consequences. Furthermore, the underlying mechanisms of action are presented and the possibility of nutritional intervention is discussed.

\section{Methods}

A literature search of the past 15 years was conducted using Medline and selected papers. The most important immunological techniques include enzyme linked immunosorbent assay techniques to measure circulating cytokine protein concentrations and quantitative polymerase chain reaction to measure mRNA for various cytokines. Flow cytometry was used to identify lymphocyte subpopulations using monoclonal antibodies. Lymphocyte function was estimated by lymphocyte proliferative responses, and cytotoxic activities were measured by assay of ${ }^{51} \mathrm{Cr}$ release.

\section{Effects of acute exercise}

EFFECT OF ACUTE EXERCISE ON LYMPHOCYTE FUNCTION

In relation to acute exercise, there are several consistent patterns that emerge with regard to leucocyte subpopulations in the blood. The neutrophil concentration increases during
An acute bout of exercise induces mobilisation of all lymphocyte subpopulations to the blood. After intense long duration exercise, the concentrations of all lymphocyte sub$\mathrm{T}$ cells are inhibited, and the local production of secretory IgA in the mucosa is inhibited. The neutrophils increase in response to exercise and continue to increase in the period after exercise. populations decline, the function of NK and 


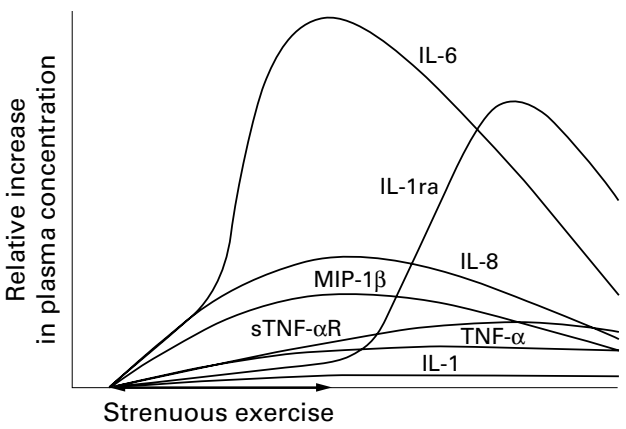

Figure 1 Effect of strenuous exercise on the levels of interleukin (IL)-6, interleukin-1 receptor antagonist

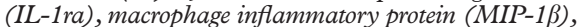
$I L-8$, soluble tumour necrosis factor-a receptor ( $T N N F-a R)$, $T N F-a$, and $I L-1$. The relative increase in plasma concentrations of cytokines during and after exercise is shown.

exercise and continues to increase after exercise. ${ }^{7}$ The lymphocyte concentration increases during exercise and falls below values found before exercise after intense long duration exercise, but is not suppressed after moderate exercise. ${ }^{7}$ The increased lymphocyte concentration is due to recruitment of all lymphocyte subpopulations to the blood. Thus the CD4 T cells, CD8 T cells, CD19 B cells, CD16 natural killer (NK) cells, and CD56 NK cells increase in number during exercise and decline after intense exercise lasting at least one hour. Furthermore, after intense long duration exercise, the functions of NK and B cells are suppressed. Thus the NK cell activity (the ability of NK cells to lyse a certain number of tumour target cells) is inhibited. Furthermore, antibody production in the circulation is inhibited, and local production of secretory IgA in the mucosa is inhibited. ${ }^{89}$

EFFECT OF ACUTE EXERCISE ON CYTOKINE LEVELS Strenuous exercise induces increased levels of cytokines in the blood (fig 1). Interleukin (IL)-6 has been found to be enhanced in several studies. ${ }^{10-17}$ Thus, after a marathon, the level of IL-6 is increased 100-fold. Although initial studies suggested that the level of IL-1 was increased in response to exercise, ${ }^{18}$ recent studies using more specific assays have shown no increase or only a modest increase. Studies from our group have shown no effect of exercise on the levels of the anti-inflammatory cytokine transforming growth factor- $\beta 1$ (A D Toft, unpublished data). The concentrations of tumour necrosis factor (TNF)- $\alpha$ have been shown to increase $2-3$-fold ${ }^{17}$ after strenuous exercise. The increase in IL-6 is followed by an increase in the concentrations of the IL-1 receptor antagonist (IL-1 ra), a naturally occurring inhibitor of IL-1. ${ }^{14} 16$ Thus the level of IL-6 peaks immediately after cessation of exercise, whereas levels of IL-1ra do not increase until after exercise, peaking after about two hours.

Recent data from our group show that the circulating levels of soluble $\mathrm{TNF}-\alpha$ receptors $(s T N F-\alpha R) 1$ and 2 and the chemokines IL-8 and macrophage inflammatory protein (MIP$1 \beta)$ are also increased in response to strenuous exercise $^{17}$ (K Ostrowski and A D Toft, unpublished data). Thus exercise induces a strong anti-inflammatory response.
POSSIBLE ASSOCIATIONS BETWEEN THE CYTOKINE RESPONSE AND MUSCLE DAMAGE

Bruunsgaard et $a l^{13}$ compared concentric and eccentric ergometer bicycle exercise and found an association between increased IL- 6 level and muscle damage, as illustrated by the increase in creatine kinase. Thus the level of IL-6 increased more during the eccentric exercise, and a significant association was found between peak IL- 6 and peak creatine kinase on the subsequent days $(r=0.722 ; \mathrm{p}=0.028)$. The eccentric bicycle model results in delayed muscle damage, with peak creatine kinase levels on day four or five after exercise.

One source of IL- 6 has recently been identified. We were able to detect IL-6 mRNA in skeletal muscle biopsy specimens obtained from runners after a marathon. ${ }^{14}$ These data indicate that IL-6 is locally produced in response to strenuous exercise or exercise induced muscle damage. IL-1ra mRNA was not present in the skeletal muscle, but was expressed by blood mononuclear cells obtained after, but not before, the marathon, indicating that locally produced IL- 6 induces a systemic anti-inflammatory response.

\section{Effects of chronic exercise}

The immune function (resting levels) in athletes compared with non-athletes has more similarities than disparities, as reviewed. ${ }^{19}$ Natural immunity may be slightly increased, whereas neutrophil function has been reported to be slightly suppressed. The adaptive immune system (resting state) in general seems to be largely unaffected by intensive and prolonged exercise training. ${ }^{20-22}$ The innate immune system appears to respond differentially to the chronic stress of intensive exercise, with NK cell activity tending to be enhanced while neutrophil function is suppressed. ${ }^{23-26}$

Natural immunity (resting levels of NK cell activity) is often found to be enhanced in athletes compared with non-athletes. Most other immune parameters do not differ between athletes and non-athletes, when measured at rest.

\section{Clinical consequences}

An important question is whether the exercise induced changes in concentrations of lymphocytes in the circulating pool, the proportional distribution of lymphocyte subpopulations, and the function of these cells are of clinical significance, especially with respect to resistance to infectious disease and malignancy.

Based on anecdotal information, a general feeling has been that, whereas regular training promotes resistance to upper respiratory tract infection (URTI), severe exertion, especially when coupled with mental stress, places athletes at increased risk of URTI. The epidemiological studies on exercise and URTI are based on self reported symptoms rather than clinical verification. ${ }^{27}{ }^{28}$ The link between exercise associated immune changes and sensitivity to infection may be explained by the so called "open 
window" of altered immunity. We have previously hypothesised that viruses and bacteria may gain a foothold, increasing the risk of subclinical and clinical infection. ${ }^{29}$ However, it remains to be shown whether athletes displaying the most extreme immunosuppression after heavy exertion are those that contract an infection within the following one to two weeks.

According to the immune surveillance theory, it is to be expected that moderate exercise protects against malignancy, whereas exhaustive exercise is linked with increased cancer risk. Results are accumulating that support the idea that exercise protects against colon cancer and breast cancer, ${ }^{30}{ }^{31}$ whereas there is little or no published evidence that strenuous exercise is associated with increased risk of cancer. Furthermore, although immune mechanisms may be important mediators of the protective exercise effect, this remains to be shown.

\section{Mechanisms of action}

NEUROENDOCRINOLOGICAL MECHANISMS

The mechanisms underlying exercise associated immune changes are multifactorial and include neuroendocrinological factors such as adrenaline (epinephrine), noradrenaline (norepinephrine), growth hormone, and cortisol. ${ }^{32} 33$ The concentrations of these hormones increase during exercise and return to original values shortly after, but they also seem to exert effects on lymphocytes and neutrophils during the recovery period. Studies in which hormones were infused, hormone receptors were blocked by drugs, or hormone production was inhibited by epidural blockade in relation to physical stress contribute to our understanding of the mechanisms of action. Based on these studies, we have proposed a model (fig 2) for the possible roles of stress hormones in mediating exercise induced immune changes during and after exercise. Adrenaline and to a lesser degree noradrenaline are responsible for acute exercise effects on lymphocyte dynamics, including exercise effects on NK cell activity and T cell function. Increases in growth hormone and catecholamines mediate the acute effects on neutrophils, whereas cortisol exerts its effects within a time lag of at least two hours and therefore may help to maintain the lymphopenia and neutrocytosis only after long term exercise. ${ }^{32}$ The role of $\beta$-endorphins is less clear, but we do not believe that they play an important role in the immediate recruitment of NK cells to the blood.

Adrenaline and to a lesser degree noradrenaline mediate the acute exercise effects on lymphocytes. Increases in growth hormone and catecholamines mediate the effect on neutrophils.

METABOLIC MECHANISMS

Altered protein metabolism during exercise may in principle help to explain exercise related immune changes. As a consequence, supplementation with nutrients such as glutamine,
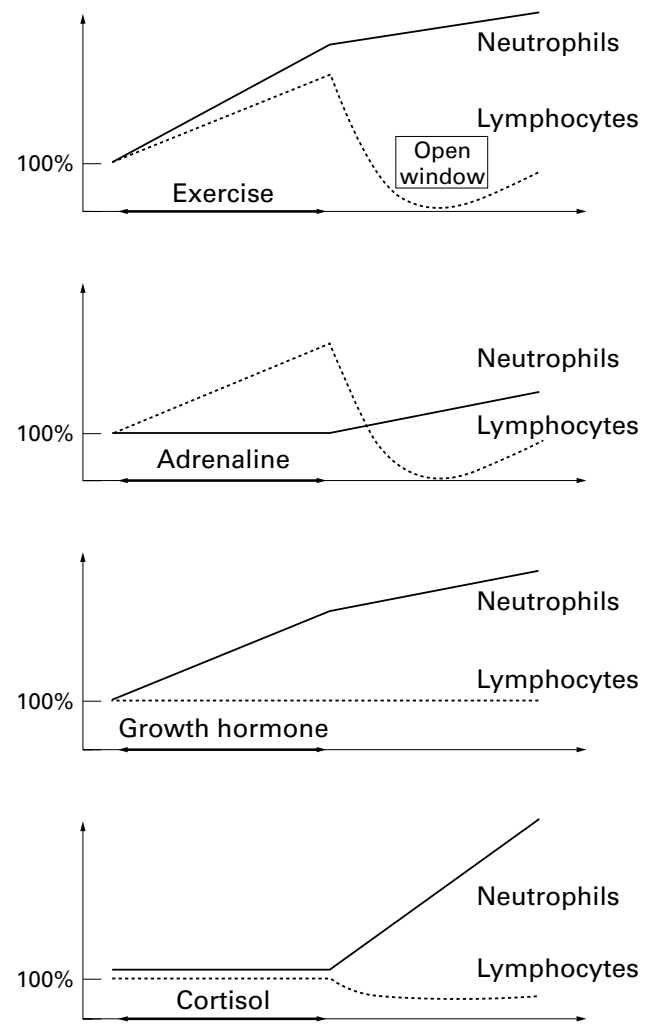

Figure 2 Model of the proposed neuroendocrinological mechanisms underlying the exercise induced "open window" in the immune system. The figures show the effect of strenuous exercise and the suggested effects of adrenaline, growth hormone, and cortisol on the relative concentrations of lymphocytes and neutrophils.

carbohydrate, antioxidants, or prostaglandin inhibitors may in principle influence exercise associated changes in immune function. ${ }^{32}$

\section{Glutamine}

It has been established that glutamine is an important fuel for lymphocytes and macrophages. Several lines of evidence suggest that it is used at a very high rate by these cells, even when they are quiescent. ${ }^{34}$ It has been proposed that the glutamine pathway in lymphocytes may be under external regulation, partly because of the supply of glutamine itself. ${ }^{35}$ Glutamine stimulates in vitro lymphocyte proliferation, lymphokine activated killer cell activity, and cytokine production. ${ }^{36} 37$

Skeletal muscle is the major tissue involved in glutamine production and it is known to release glutamine into the bloodstream at a high rate. It has been suggested that the skeletal muscle plays a vital role in maintenance of the key process of glutamine utilisation in the immune cells. Consequently, the activity of the skeletal muscle may directly influence the immune system. It has been hypothesised (the glutamine hypothesis) that during intense physical exercise, or in association with surgery, trauma, burn, and sepsis, the demands on muscle and other organs for glutamine is such that the lymphoid system may be forced into a glutamine debt, which temporarily affects its function. Thus factors that directly or indirectly influence glutamine synthesis or release could theoretically influence the function of 
lymphocytes and monocytes. ${ }^{34} 3839$ After intense long term exercise and other physical stress disorders, the glutamine concentration in plasma declines. ${ }^{40-45}$ Furthermore, low glutamine levels have been described in athletes with overtraining syndrome. ${ }^{44-48} \mathrm{Op}$ timal lymphocyte proliferation is dependent on the presence of glutamine, but there are no published data showing that glutamine supplementation restores impaired immune function after exercise. The critical question therefore is not whether concomitant decreased plasma glutamine concentration and lymphocyte function occur after intense exercise, but whether a causal relation exists. In two recent placebo controlled glutamine intervention studies, ${ }^{49}{ }^{50}$ it was found that glutamine abolished the decline in plasma glutamine after exercise without influencing the immunosuppression found. Thus these studies did not support the hypothesis that the decline in immune function after exercise is caused by a decrease in plasma glutamine concentration.

Carbohydrate and immune function

Earlier research has established that a reduction in blood glucose levels is linked to hypothalamic-pituitary-adrenal activation, an increased release of adrenocorticotrophic hormone and cortisol, increased plasma growth hormone, decreased insulin, and a variable effect on blood adrenaline level. ${ }^{2}$ Given the link between stress hormones and immune responses to prolonged and intensive exercise, ${ }^{32}$ carbohydrate compared with placebo ingestion should maintain plasma glucose concentrations, attenuate increases in stress hormones, and thereby diminish changes in immunity. This hypothesis has been tested in a number of studies by Nieman et $a P^{1-53}$ using double blind, placebo controlled randomised designs. Carbohydrate beverage ingestion before, during (about 1 litre/hour), and after 2.5 hours of exercise was associated with higher plasma glucose levels, an attenuated cortisol and growth hormone response, fewer perturbations in blood immune cell counts, lower granulocyte and monocyte phagocytosis and oxidative burst activity, and a diminished proinflammatory and anti-inflammatory cytokine response. Overall, the hormonal and immune responses to carbohydrate compared with placebo ingestion were diminished. Some immune variables were affected slightly by carbohydrate ingestion-for example, granulocyte and monocyte function-while others were strongly influenced-for example, plasma cytokine concentrations and blood cell counts.

The clinical significance of these carbohydrate induced effects on the endocrine and immune system awaits further research. At this point, the data indicate that athletes ingesting carbohydrate beverages before, during, and after prolonged and intensive exercise should experience lowered physiological stress. Research to determine whether carbohydrate ingestion improves host protection against infection in endurance athletes during periods of intensified training or after competitive endurance events is warranted.
Lipids

It has been suggested that, if the polyunsaturated fatty acid (PUFA) profile of $n-6$ and $n-3$ is shifted in favour of $n-6$, this will result in increased production of prostaglandin $\left(\mathrm{PGE}_{2}\right)$ and leukotriene $\left(\mathrm{LT}_{4}\right)$. The arachidonic acid derived eicosanoids $\mathrm{PGE}_{2}$ and $\mathrm{LT}_{4}$ modulate the production of proinflammatory and immunoregulatory cytokines. ${ }^{54}$ The $n-3$ PUFAs eicosapentaenoic acid and docosahexaenoic acid, both found particularly in fish oils, suppress the production of arachidonic acid derived eicosanoids. ${ }^{55}$ Eicosapentaenoic acid is a substrate for the synthesis of an alternative family of eicosanoids, $\mathrm{PGE}_{3}$ and $\mathrm{LT}_{5}$, whereas arachidonic acid is a substrate for $\mathrm{PGE}_{2}$ and $\mathrm{LT}_{4}{ }^{56}$

$\mathrm{PGE}_{2}$ suppresses the cellular immune system. During stress conditions, $n-3$ PUFA may counteract latent immunosuppression mediated by increasing $\mathrm{PGE}_{2}$ production, which in contrast appears to be further enhanced by intake of $n-6$ PUFA. Under conditions of hypermetabolism, n-3 PUFA therefore potentially acts to reduce the incidence of new infections.

In animal studies, the stress response after application of endotoxin, IL-1, or TNF- $\alpha$ was reduced when the animals were pretreated with n-3 PUFA (fish oil). The diet rich in $n-3$ PUFA caused reduced catabolism, reduced febrile reaction, decreased eicosanoid production, and improved survival rate. ${ }^{57}$

The possible interaction between intense acute exercise, known to suppress the immune system, ${ }^{58}$ and PUFA was examined in inbred female $\mathrm{C} 57 \mathrm{BI} / 6$ mice. ${ }^{59}$ The animals received either a natural ingredient diet or a diet supplemented with various oils such as beef tallow, safflower, fish oil, or linseed oil for an eight week period. In the group receiving 18:3 (n-3 PUFA) linseed oil, post-exercise immunosuppression of the IgM plaque forming cell response was abolished. The mechanism underlying the absence of exercise induced immunosuppression in animals fed linseed oil may be that linseed oil decreases the $n-6 / n-3$ ratio and thereby diminishes the $\mathrm{PGE}_{2}$ level after intense exercise. Thereby, the prostaglandin mediated immunosuppression may be abolished.

Thus the effect of linseed oil may be ascribed to a link between a diet rich in n-3 PUFA and abolition of prostaglandin related immunosuppression. In support of this hypothesis, it has been shown that, when the $\mathrm{PGE}_{2}$ production was inhibited by the prostaglandin inhibitor indomethacin, exercise induced suppression of the NK cell activity and B cell function was partly abolished. ${ }^{60} 61$

Dietary fats that are rich in $n-3$ PUFA have the potential to alter cytokine production. Most studies provide evidence that feeding plant or fish oils rich in n-3 PUFA alters the ex vivo production of TNF- $\alpha$, IL-1, IL-2, and IL-6, ${ }^{62-65}$ but contradictory observations do exist. Human studies provide more consistent data: several studies have shown that supplementation of the diet of healthy volunteers results in reduced production of IL-1, IL-6, TNF- $\alpha$, and IL-2 by peripheral blood 
mononuclear cells in vitro. ${ }^{66}$ In one study, supplementation resulted in decreased levels of IL-2 and IL-6 in vivo. ${ }^{67} 68$

Our group has recently investigated whether dietary supplementation with $n-3$ PUFA before participation in strenuous exercise influences the production of proinflammatory and anti-inflammatory cytokines. No differences were found between the supplementation group and the control group (A D Toft, unpublished data).

\section{Antioxidants}

It has been suggested that antioxidant vitamins may influence exercise induced immune activation by neutralising the reactive species produced by neutrophils during phagocytosis. ${ }^{69} 70$ Peters $e t a l^{71}$ evaluated the effect of vitamin C on the incidence of URTI during the two week period after the $90 \mathrm{~km}$ Comrades Ultramarathon using a double blind randomised design. The incidence of URTI was $68 \%$ in the placebo group, which was significantly more than in the vitamin $\mathrm{C}$ supplementation group, in which only $33 \%$ reported URTI when taking a $600 \mathrm{mg}$ vitamin $\mathrm{C}$ supplementation daily for three weeks before the race. In another study, Peters et $a l^{72}$ found that vitamin A supplementation had an insignificant effect on the incidence of URTI in marathon runners. Only one study ${ }^{73}$ has evaluated the effect of vitamin $C$ on acute exercise induced changes in lymphocyte function and stress hormone levels. Supplementation with vitamin C did not influence leucocyte subsets, NK cell activity, lymphocyte proliferative response, granulocyte phagocytosis and activated burst, catecholamines, or cortisol.

\section{Conclusion}

During an acute bout of exercise, immunocompetent cells are mobilised to the circulation. Thus both the neutrophils and all lymphocyte subpopulations are recruited to the blood circulation. However, after strenuous exercise, the lymphocyte count declines below baseline, whereas the concentration of neutrophils continues to increase. Also, the levels of secretory $\operatorname{IgA}$ in the mucosa decrease. In response to exercise, a pronounced increase in both proinflammatory and anti-inflammatory cytokines is found. All these factors indicate a strong inflammatory response during strenuous exercise. Thus exercise produces concomitant inflammation and immune impairment.

The clinical consequences of repeated hard exercise are subclinical and clinical infections. The explanation may be that virus and bacteria gain a foothold after exercise by the time of the "open window" with altered immunity. The underlying mechanisms are multifactorial and include both neuroendocrinological and metabolic factors. Nutritional supplementation may in principle protect against the increased risk of infection in the recovery period after strenuous exercise. Carbohydrate supplementation has been shown to moderate the exercise induced immune changes, but the clinical significance remains to be shown. Thus it is premature to offer advice on nutrition to athletes from an immunological point of view.

1 Fitzgerald L. Exercise and the immune system. Immunol Today 1988;9:337-9.

2 Nieman DC, Pedersen BK. Exercise and immune function: recent development. Sports Med 1999;27:73-80.

3 Nieman DC. Exercise and resistance to infection. Can $\mathcal{f}$ Physiol Pharmacol 1998;76:573-80.

4 Hoffman-Goetz L, Husted J. Exercise and cancer: do the biology and epidemiology correspond? Exercise and Immunology Review 1995;1:81-96.

5 Brines R, Hoffman-Goetz L, Pedersen BK. Can you exercise to make your immune system fitter? Immunol Today 1996;17:252-4.

6 Pedersen BK, Hoffman-Goetz L. Exercise and the immune system: regulation, integration and adaptation. Physiol Rev 2000; in press.

7 McCarthy DA, Dale MM. The leucocytosis of exercise. A review and model. Sports Med 1988;6:333-63.

8 Pedersen BK. Pedersen BK, eds. Exercise immunology. Pedersen BK. Pedersen BK, eds. Exercise imm
Austin, TX: RG Landes Bioscience, 1997:1-206.

Austin, TX: RG Landes Bioscience, 1997:1-206.
9 Pedersen BK, Nieman DC. Exercise immunology: integraPedersen BK, Nieman DC. Exercise immunology: inte
tion and regulation. Immunol Today 1998;19:204-6.

10 Northoff H, Berg A. Immunologic mediators as parameters Northoff H, Berg A. Immunologic mediators as parameters
of the reaction to strenuous exercise. Int $\mathcal{F}$ Sports Med 1991; 12(suppl 1):S9-15.

11 Ullum H, Haahr PM, Pedersen BK, et al. Bicycle exercise enhances plasma IL-6 but does not change IL-1alpha, IL-1beta, IL-6, or TNF-alpha pre-mRNA in BMNC. $\mathscr{f}$ Appl Physiol 1994;77:93-7.

12 Sprenger H, Jacobs C, Gemsa D, et al. Enhanced release of cytokines, interleukin-2 receptors, and neopterin after ong-distance running. Clin Immunol Immunopathol 1992; 63:188-95.

13 Bruunsgaard H, Galbo H, Pedersen BK, et al. Exerciseinduced increase in interleukin-6 is related to muscle damage. F Physiol (Lond) 1997;499:833-41.

14 Ostrowski K, Rohde T, Pedersen BK, et al. Evidence that IL-6 is produced in skeletal muscle during intense long-term muscle activity. $\mathcal{F}$ Physiol (Lond) 1998;508:94953.

15 Rohde T, MacLean DA, Pedersen BK, et al. Prolonged submaximal eccentric exercise is associated with increased levels of plasma IL-6. Am f Physiol 1997;273:E85-91.

16 Ostrowski K, Hermann C, Pedersen BK, et al. A trauma-like elevation in plasma cytokines in humans in response to treadmill running. I Physiol (Lond) 1998;508:949-53.

17 Ostrowski K, Rohde T, Pedersen BK, et al. Pro- and anti-inflammatory cytokine balance in strenuous exercise in humans. $\mathcal{F}$ Physiol (Lond) 1999;515:287-91.

8 Cannon JG, Kluger MJ. Endogenous pyrogen activity in human plasma after exercise. Science 1983;220:617-19.

19 Nieman DC. Hoffman-Goetz L. In: Hoffman-Goetz L, ed. Exercise and immune function. New York: CRC Press, 1996: 143-62.

20 Baj Z, Kantorski J, Lewicki R, et al. Immunological status of competitive cyclists before and after the training season. Int F Sports Med 1994;15:319-24

21 Tvede N, Steensberg J, Pedersen BK, et al. Cellular immunity in highly trained elite racing cyclists during periods of nity in highly trained elite racing cyclists during periods of
training with high and low intensity. Scand 7 Med Sci Sports 1991;1:163-6.

22 Nieman DC, Buckley KS, Nehlsen Cannarella SL, et al. Immune function in marathon runners versus sedentary controls. Med Sci Sports Exerc 1995;27:986-92.

23 Hack V, Strobel G, Weicker H, et al. PMN cell counts and phagocytic activity of highly trained athletes depend on training period. F Appl Physiol 1994;77:1731-5

24 Nieman DC, Henson DA, Nehlsen Cannarella SL, et al. Physical activity and immune function in elderly women. Med Sci Sports Exerc 1993;25:823-31.

25 Pedersen BK, Tvede N, Halkjær Kristensen J, et al. Natural killer cell activity in peripheral blood of highly trained and untrained persons. Int F Sports Med 1989;10:129-31.

26 Pyne DB, Baker MS, Weidemann MJ, et al. Effects of an intensive 12 -wk training program by elite swimmers on neutrophil oxidative activity. Med Sci Sports Exerc 1995;27: neutrop

27 Fitzgerald L. Overtraining increases the susceptibility to infection. Int $\mathcal{F}$ Sports Med 1991;12(suppl 1):S5-8.

28 Nieman DC. Exercise, upper respiratory tract infection, and Nieman DC. Exercise, upper respiratory tract infection, and
the immune system. Med Sci Sports Exerc 1994;26:128-39.

29 Pedersen BK, Ullum H. NK cell response to physical activity: possible mechanisms of action. Med Sci Sports Exerc 1994;26:140-6.

30 Hoffman-Goetz L, Hoffman-Goetz L, Husted J. In: Hoffman-Goetz L, ed. Exercise and immune function. New York: CRC Press, 1996:179-98.

31 Thune I, Brenn T, Gaard M, et al. Physical activity and the risk of breast cancer. N Engl f Med 1997;336:1269-75.

32 Pedersen BK, Bruunsgaard H, Zacho M, et al. Exerciseinduced immunomodulation: possible roles of neuroendocrine factors and metabolic factors. Int $\mathcal{F}$ Sports Med 1997; 18(suppl 1):S2-7.

33 Pedersen BK, Nieman DC. Exercise and immunology: integration and regulation. Immunol. Today 1998;19:204-6.

34 Newsholme EA. Biochemical mechanisms to explain immunosuppression in well- trained and overtrained immunosuppression in well- trained a

35 Ardawi MS, Newsholme EA. Intracellular localization and properties of phosphate-dependent glutaminase in rat mesenteric lymph nodes. Biochem f 1984;217:289-96. 
36 Rohde T, Ullum H, Pedersen BK, et al. Effects of glutamine on the immune system: influence of muscular exercise and on the immune system: influence of muscular
HIV infection. 7 Appl Physiol 1995;79:146-50.

37 Rohde T, MacLean DA, Pedersen BK. Glutamine, lymphocyte proliferation and cytokine production. Scand $\mathcal{F}$ Immunol 1996;44:648-50.

38 Newsholme EA. Psychoimmunology and cellular nutrition: an alternative hypothesis [editorial]. Biol Psychiatry 1990; 27:1-3.

39 Wallace C, Keast D. Glutamine and macrophage function. Metabolism 1992;41:1016-20.

40 Parry Billings M, Budgett R, Newsholme EA, et al. Plasma amino acid concentrations in the overtraining syndrome: possible effects on the immune system. Med Sci Sports Exerc 1992;24:1353-8.

41 Keast D, Arstein D, Morton AR, et al. Depression of plasma glutamine concentration after exercise stress and its possible influence on the immune system. Med f Aust 1995;162: 15-18.

42 Essen $\mathrm{P}$, Wernerman J, Vinnars E, et al. Free amino acids in plasma and muscle during 24 hours postoperatively: a plasma and muscle during 24 hours postop
descriptive study. Clin Physiol 1992;12:163-77.

43 Lehmann M, Huonker M, Haussinger D, et al. Serum amino acid concentrations in nine athletes before and after the 1993 Colmar ultra triathlon. Int $\mathcal{F}$ Sports Med 1995;16: $155-9$.

44 Keast D, Hoffman-Goetz L. In Hoffman-Goetz L, ed. Exer cise and immune function. New York: CRC Press, 19967: 121-42.

45 Rohde T, MacLean DA, Pedersen BK, et al. The immune system and serum glutamine during a triathlon. Eur $\mathcal{F} A p p l$ Physiol 1996;74:428-34.

46 Rowbottom DG, Keast D, Morton AR, et al. Training adaptation and biological changes among well-trained male triathletes. Med Sci Sports Exerc 1997;29:1233-9.

47 Rowbottom DG, Keast D, Morton AR. The emerging role of glutamine as an indicator of exercise stress and of glutamine as an indicator of ex.

48 Keast D, Morton AR. Long-term exercise and immune functions. In: Watson RR, Eisinger M, eds. Exercise and disfunctions. In: Wats

49 Rohde T, MacLean D, Pedersen BK. Effect of glutamine on changes in the immune system induced by repeated exercise. Med Sci Sports Exerc 1998;30:856-62.

50 Rohde T, Asp S, Pedersen BK, et al. Competitive sustained exercise in humans, lymphokine activated killer cell activity, and glutamine: an intervention study. Eur $\mathcal{F} A p p$ Physiol 1998;78:448-53.

51 Nehlsen-Canarella SL, Fagoaga OR, Nieman DC. Carbohydrate and the cytokine response to 2.5 hours of running. $f$ Appl Physiol 1997;82:1662-7.

52 Nieman DC, Henson DA, Nehlsen-Cannarella SL, et al. Carbohydrate affects natural killer cell redistribution but not activity after running. Med Sci Sports Exerc 1997;29. 1318-24

53 Nieman DC, Fagoaga OR, Nehlsen-Canarella SL, et al. Carbohydrate supplementation affects blood granulocyte and monocyte trafficking but not function after 2.5 hours of running. F Appl Physiol 1997;82:1385-94.

54 Calder PC. n-3 polyunsaturated fatty acids and cytokine production in health and disease. Ann Nutr Metab 1997;41: production 2034 .

55 Fritsche KL, Alexander DW, Huang SC, et al. Maternallysupplied fish oil alters piglet immune cell fatty acid profile and eicosanoid production. Lipids 1993;28:677-82.
56 Calder PC. Fat chance of immunomodulation. Immunol Today 1998;19:244-7.

57 Johnson JA3, Griswold JA, Muakkassa FF. Essential fatty acids influence survival in sepsis. $\mathcal{F}$ Trauma 1993;35:12831

58 Hoffman-Goetz L, Pedersen BK. Exercise and the immune system: a model of the stress response? Immunol Today 1994;15:382-7.

59 Benquet C, Krzystyniak K, Guertin F, et al. Modulation of exercise-induced immunosuppression by dietary polyunsaturated fatty acids in mice. $\mathcal{F}$ Toxicol Environ Health 1994; 43:225-37.

60 Tvede N, Heilmann C, Pedersen BK, et al. Mechanisms of B-lymphocyte suppression induced by acute physical exercise. F Clin Lab Immunol 1989;30:169-73.

61 Pedersen BK, Tvede N, Halkjaer Kristensen $\mathrm{J}$, et al. Indomethacin in vitro and in vivo abolishes post-exercise suppression of natural killer cell activity in peripheral blood. Int F Sports Med 1990;11:127-31.

62 Billiar TR, Bankey PE, Cerra FB, et al. Fatty acid intake and Kupffer cell function: fish oil alters eicosanoid and monokine production to endotoxin stimulation. Surgery 1988;104: 343-9.

63 Hardardottir I, Kinsella JE. Increasing the dietary (n-3) to $(n-6)$ polyunsaturated fatty acid ratio increases tumor necrosis factor production by murine resident peritoneal macrophages without an effect on elicited peritoneal macmacrophages without an effect on elicit

64 Jolly CA, Jiang YH, McMurray DN, et al. Dietary (n-3) polyunsaturated fatty acids suppress murine lymphoproliferation, interleukin-2 secretion, and the formation of diacylglycerol and ceramide. $\mathcal{F}$ Nutr 1997;127:37-43.

65 Tappia PS, Grimble RF. Complex modulation of cytokine induction by endotoxin and tumour necrosis factor from peritoneal macrophages of rats by diets containing fats of different saturated, monounsaturated and polyunsaturated fatty acid composition. Clin Sci (Colch) 1994;87:173-8.

66 Endres S, Ghorbani R, Weber PC, et al. The effect of dietary supplementation with $\mathrm{n}-3$ polyunsaturated fatty acids on the synthesis of interleukin- 1 and tumor necrosis factor by mononuclear cells. N Engl F Med 1989;320:265-71.

67 Endres S, Meydani SN, Dinarello CA, et al. Dietary supplementation with $\mathrm{n}-3$ fatty acids suppresses interleukin-2 production and monon

68 Meydani SN, Endres S, Gorbach SL, et al. Oral (n-3) fatty acid supplementation suppresses cytokine production and lymphocyte proliferation: comparison between young and older women. 7 Nutr 1991;121:547-55.

69 Babior BM. Oxidants from phagocytes: agents of defense and destruction. Blood 1984;64:959-66.

70 Hemila H. Vitamin C and the common cold. Br 7 Nutr 1992;67:3-16.

71 Peters EM, Goetzsche JM, Noakes TD, et al. Vitamin C supplementation reduces the incidence of postrace symptoms of upper-respiratory-tract infection in ultramarathon runners. Am 7 Clin Nutr 1993;57:170-4.

72 Peters EM, Cambell A, Pawley L. Vitamin A fails to increase resistance to upper respiratory in distance runners. South African fournal of Sports Medicine 1992;7:3-7.

73 Nieman DC, Henson DA, Nehlsen-Canarella SL, et al. Vitamin C supplementation does not alter the immune response to 2.5 hours of running. Int $\mathcal{F}$ Sport Nutr 1997; $173-84$.

\section{Take home message}

(a) Strenuous exercise induces immune changes, including lymphopenia, neutrophilia, and elevated levels of proinflammatory cytokines.

(b) Strenuous exercise is associated with decreased resistance to upper respiratory tract infections such as the common cold in the days after exercise, whereas moderate exercise seems to offer some protection against infections.

(c) With regard to nutritional supplementation, only carbohydrate ingestion before, during, and after strenuous exercise has been experimentally shown to moderate exercise induced immunosuppression.

\section{True or false?}

1 The lymphocyte count increases during exercise and is suppressed in the period after exercise.

2 Glutamine supplementation abolishes the immunosuppression found after exercise.

3 There is epidemiological evidence that exercise protects against colon cancer.

4 There is epidemiological evidence that exercise protects against rectum cancer.
5 Neuroendocrinological factors do not play a role in exercise immunology.

\section{(Answers p 318.)}

\section{Essay question}

Describe the effect of exercise on cytokine production. What is the name of the cytokine that increases the most? 